\title{
Research on the Propagation Pattern of 3-D Initial Crack in Rock-like Material under Uniaxial Tension
}

\author{
L. Yang ${ }^{1,2, a}$, Y. Jiang ${ }^{1, b}$, S. $\mathrm{Li}^{2, \mathrm{c}}$ and B. $\mathrm{Li}^{1, \mathrm{~d}}$ \\ ${ }^{1}$ Faculty of Engineering, Nagasaki University, Nagasaki City, 852-8521, Japan \\ ${ }^{2}$ Geotechnical \& Structural Engineering Research Center, Shandong University, \\ Jinan City, 250061, P.R. China \\ asdylei@gmail.com, ${ }^{b}$ jiang@nagasaki-u.ac.jp, ${ }^{c}$ lishucai@sdu.edu.cn, ${ }^{d}$ libo@nagasaki-u.ac.jp
}

Keywords: 3-D crack, propagation pattern, uniaxial tension, wrapping wing crack, stress intensity factor, rock-like material, numerical simulation.

Abstract. Experiments and numerical simulations were conducted to investigate the propagation patterns of pre-existing 3-D elliptical crack in rock-like material under uniaxial tension. Research results demonstrated that as the load was increased, the wrapping wing cracks initiated near the front of long axis of crack, with the lateral growth of initial crack close to the front of short axis. A distortion surface formed from the growth of initial crack leaded to the macro fracture of samples. The distribution laws of mixed-mode stress intensity factor (SIF) along crack contour were derived with FRANC3D, and propagation process of 3-D crack under tension was simulated. It was concluded that propagation patterns of 3-D crack under tension were quite different from compressive cases, mainly in aspects of growth orientation and velocity.

\section{Introduction}

Fractured Rock mass is an important engineering medium encountered in many geological objects. Flaws like cracks in rock mass would result in redistribution and concentration of stress. Failure of rock mass is usually preceded by processes of initiation and accumulation of new cracks and growth of existing ones [1]. This constitutes the necessity to study the propagation patterns of cracks and its influence on mechanical properties of rock mass.

In recent years, studies on mechanisms of 3-D crack propagation have attracted much attention. A.V. Dyskin et al [1] analyzed the influence of shape and location of initial cracks on their growth in compression, and proposed the effect of intrinsic limits of wrapping wing cracks. A.V. Dyskin et al [2] developed a model of 3-D wing crack growing in compression from a disk-like initial crack. In uniaxial compression tests on PMMA samples by M. Adams and G. Sines [3], the lateral growth of initial elliptical crack (developing of squamous microcracks) was observed. Experiments on 3-D surface crack in PMMA and marble specimens were conducted by R.H.C. Wong et al [4] to investigate the influence of depth and inclination of crack on its propagation patterns. Z.Z. Liang and C.A. Tang [5] investigated the failure process of heterogeneous rock with the RFPA3D code, and established a 3-D soften model based on statistical mechanics.

Most previous studies focused on mechanisms of crack growth in compression. The propagation process of 3-D crack in rock mass under tension is still not understood sufficiently. In the present study, experiments and numerical simulations are performed to investigate the propagation patterns of 3-D internal crack in rock-like material under uniaxial tension.

\section{Experimental Studies}

Experimental Techniques and Preparation. To simulate the physico-mechanical properties of sandstone, a new kind of rock-like mortar material mixed with cement, sand and water was developed, and its physico-mechanical parameters are listed in Table 1.

Shape and size of end-enlarged sample containing single pre-existing crack is showed in Fig. 1 . The size of sample is: $W_{0} \times H_{0} \times T_{0}=100 \mathrm{~mm} \times 200 \mathrm{~mm} \times 50 \mathrm{~mm}, H_{1}=H_{2}=50 \mathrm{~mm}, W \times H \times T=$ 
$50 \mathrm{~mm} \times 100 \mathrm{~mm} \times 50 \mathrm{~mm}$. Size of elliptical crack is: $2 a \times 2 b=20 \mathrm{~mm} \times 10 \mathrm{~mm}$. To evaluate the influence of inclination of crack, inclination angles $\alpha$ (inclination to loading axis) were set to $30^{\circ}, 45^{\circ}$, $60^{\circ}, 75^{\circ}$ and $90^{\circ}$, respectively. Uniaxial tensile tests were carried out in a displacement-controlled mode at a rate of $0.002 \mathrm{~mm} / \mathrm{s}$ with a servo-control rigid loading frame.

Table 1. Physico-mechanical parameters of mortar

\begin{tabular}{|c|c|c|c|c|c|c|}
\hline Parameters & Density & $\begin{array}{c}\text { Compressive } \\
\text { strength }\end{array}$ & $\begin{array}{c}\text { Tensile } \\
\text { strength }\end{array}$ & $\begin{array}{c}\text { Modulus of } \\
\text { elasticity }\end{array}$ & $\begin{array}{c}\text { Poisson's } \\
\text { ratio }\end{array}$ & $\begin{array}{c}\text { Fracture } \\
\text { toughness }\end{array}$ \\
\hline Units & $\rho\left[\mathrm{g} / \mathrm{cm}^{3}\right]$ & $\sigma_{\mathrm{c}}[\mathrm{MPa}]$ & $\sigma_{\mathrm{t}}[\mathrm{MPa}]$ & $E[\mathrm{GPa}]$ & $v$ & $K_{\mathrm{IC}}\left[\mathrm{MPa} \cdot \mathrm{m}^{0.5}\right]$ \\
\hline Mortar & 2.3 & 35.5 & 2.8 & 17.9 & 0.19 & 0.51 \\
\hline
\end{tabular}

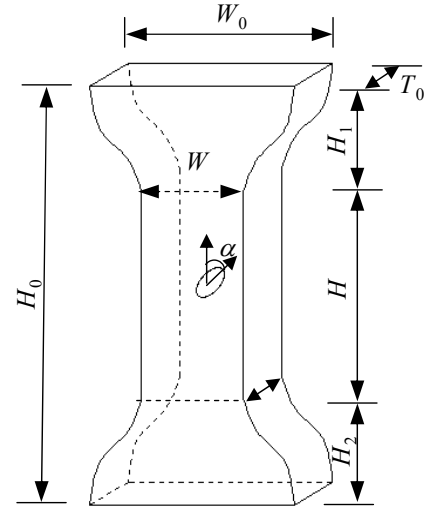

Fig. 1. Shape and size of sample

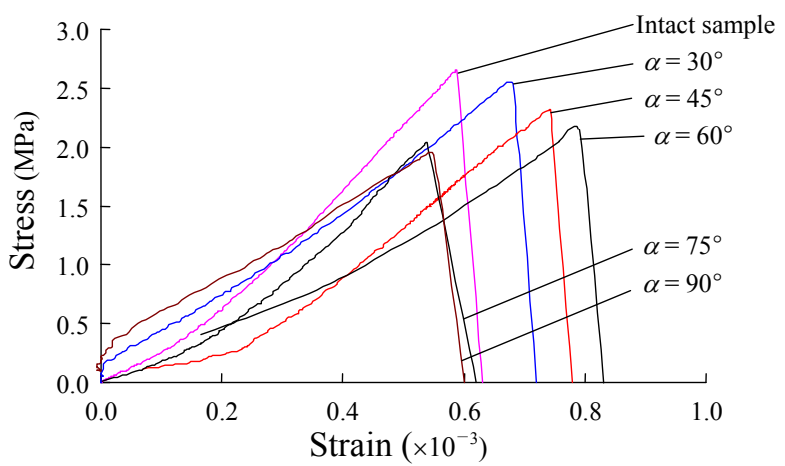

Fig. 2. Uniaxial tensile stress-strain curves of different samples

Experimental Results. Uniaxial tensile stress-strain curves of different samples are showed in Fig. 2. It is derived that curve shape in tensile condition is quite different from that in compression. Process of loading and deforming of rock-like samples can be divided into 4 stages: crack opening stage, elastic deforming stage, elastic \& plastic deforming stage and failure stage. Elastic deformation takes most proportion of the whole. Unlike compressive case, tensile failure stage is abrupt, with the stress reducing to 0 rapidly. Inclination angle $\alpha$ of initial crack has obvious influence on sample strength. As $\alpha$ increases, the strength of sample decreases gradually. This fact is due to the reason that stress component normal to crack plane increased with increase of $\alpha$.

Failure status of samples (Fig. 3) indicates that fracture happened in the middle of sample. Fracture trace line is at a smaller angle to horizontal plane than the inclination of initial crack, and growth trace of wing crack is observed on the fracture section. Trace line is winding, and fracture section is tortuous. This fact is due to the heterogeneous of mortar material.

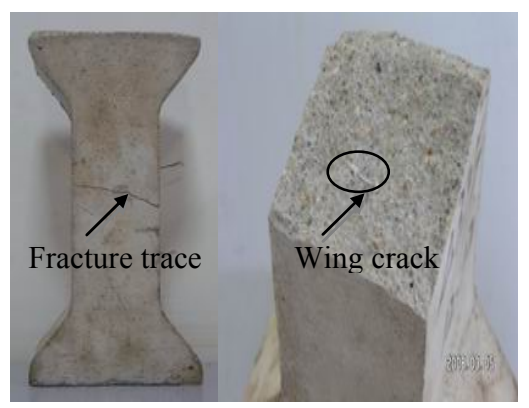

Fig. 3. Failure status of sample (e.g. $\alpha=45^{\circ}$ case)

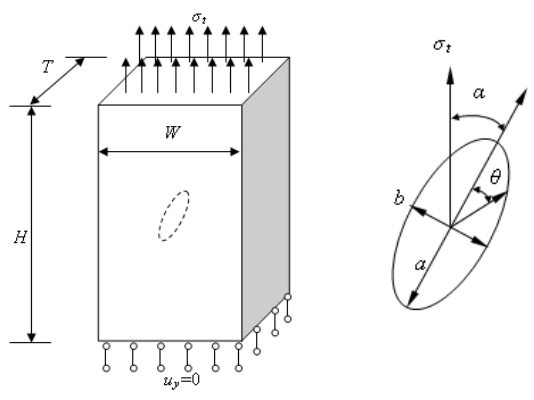

Fig. 4. Numerical model and crack location

\section{Numerical Simulation}

Numerical Model. FRANC3D (FRacture ANalysis Code-3D) developed by Cornell Fracture Group was used to investigate the distribution of Stress Intensity Factor (SIF) along the front of initial crack, 
and the propagation process of crack under uniaxial tensile stress. The uniform-cross segment $(W \times H$ $\times T=50 \mathrm{~mm} \times 100 \mathrm{~mm} \times 50 \mathrm{~mm}$ ) of experimental sample was chosen as numerical model (Fig. 4), and inclination angle of initial crack $\alpha$ was $15^{\circ}, 30^{\circ}, 45^{\circ}, 60^{\circ}, 75^{\circ}$ and $90^{\circ}$, respectively. Symbol $\theta$ represents the angle between radial lines and long axis of crack. Physico-mechanical parameters of model are listed in Table 1. Uniaxial tensile stress $\sigma_{\mathrm{t}}$ was set to $2 \mathrm{MPa}$ to ensure sufficient growth of crack.

SIF Distribution along Crack Contour. The normalization of mode I, II and III SIF for all cracks was performed by using Eq. (1) and (2), where $a, b$ represented the semi-length of long and short axis of initial crack, respectively.

$$
\begin{aligned}
& K_{R}=\sigma_{t} \sqrt{\frac{\pi a}{Q}} \\
& Q=1+1.464(a / b)^{1.65}
\end{aligned}
$$

Distribution curves of normalized mode I, II and III SIFs are showed in Fig. 5, 6 and 7. It is demonstrated that $K_{\mathrm{I}}$ and $K_{\mathrm{II}}$ curves are symmetric to the line of $\theta=180^{\circ}$ which represents long axis of crack, while $K_{\text {III }}$ curves are antisymmetric to $\theta=180^{\circ}$ line. Fig. 5 indicates that $K_{\text {I }}$ increases when $\theta$ increases from $0^{\circ}$ to $90^{\circ}$. Maximum value of $K_{\mathrm{I}}$ locates in end points of short axis, and minimum value locates in end points of long axis. Inclination angle $\alpha$ has obvious influence on $K_{\mathrm{I}}$ values. With the increase of $\alpha$, values of $K_{\mathrm{I}}$ increases gradually and reaches maximum when $\alpha=90^{\circ}$ (horizontal initial crack).

$K_{\text {II }}$ curves are linear in the range of $0^{\circ}<\theta<180^{\circ}$, and antisymmetric to line of $\theta=90^{\circ}$, as showed in Fig. 6. When $\theta$ grows from $0^{\circ}$ to $90^{\circ}, K_{\mathrm{II}}$ decreases to 0 gradually. Inclination angle $\alpha$ affects the distribution of $K_{\mathrm{II}}$ distinctly. $K_{\mathrm{II}}$ increases with the increase of $\alpha$ in the range of $0^{\circ}<\alpha<45^{\circ}$, but decreases when $\alpha$ exceeded $45^{\circ}$.

Fig. 7 demonstrates that $K_{\text {III }}$ curves are parabolic with maximum values located in end points of short axis. Influence of inclination angle $\alpha$ on $K_{\text {III }}$ distribution is generally the same with $K_{\text {II }}$ case. That is to say that absolute values of $K_{\text {III }}$ reaches maximum when $\alpha=45^{\circ}$

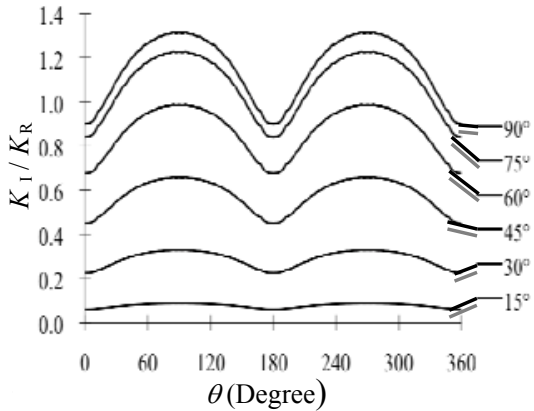

Fig. 5. Distribution of normalized $K_{\mathrm{I}}$

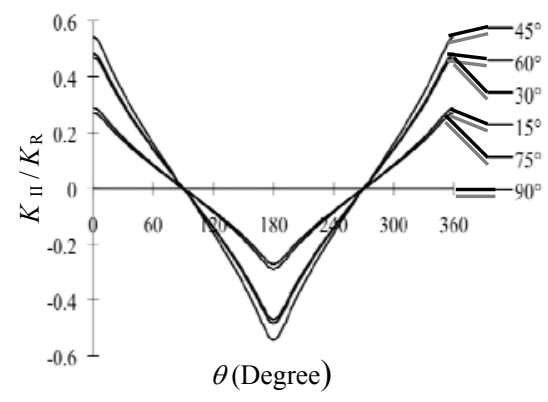

Fig. 6. Distribution of normalized $K_{\mathrm{II}}$

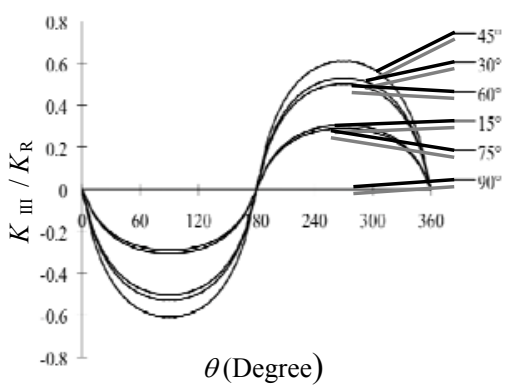

Fig. 7. Distribution of normalized $K_{\mathrm{III}}$

Propagation Process of Initial Crack. For this mixed-mode fracture problem, determination of fail/safe condition or prediction of internal crack growth rate can be achieved by the well-known energy release rate fracture criteria, which is applicable for 3-D cases. The numerical simulation of propagation process of initial crack under tensile stress is showed in Fig. 8. The propagation of wing crack and lateral growth due to tensile stress are observed obviously. Fig. 8a shows the initial state of pre-existing elliptic crack. When uniaxial stress exceeds initiation stress, wing crack develops in the front of long axis of crack, and grows in the direction approximately perpendicular to initial crack plane (Fig. 8b). At the same time, lateral growth in the front of short axis is observed, and its growth rate is even greater than that of wing crack. Unlike wing crack, direction of lateral growth is along the initial crack plane. With the increase of stress, growth rate of initial crack increases correspondingly (Fig. 8c). When wing crack propagates to some extent, its growth direction changes to horizontal direction gradually (Fig. 8d). However, direction of later growth keeps invariant. The wrapping wing 
crack and later growth of initial crack forms a spatial distortion plane (Fig. 8e), which finally results in the overall fracture of sample.

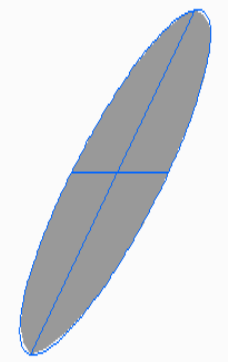

(a)

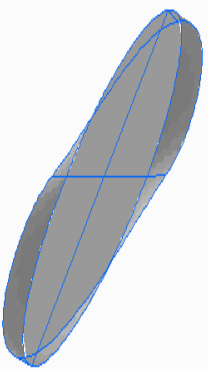

(b)

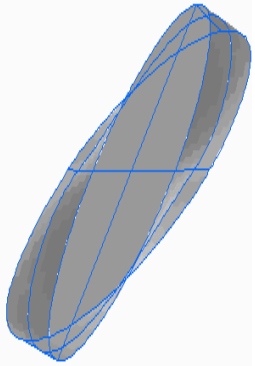

(c)

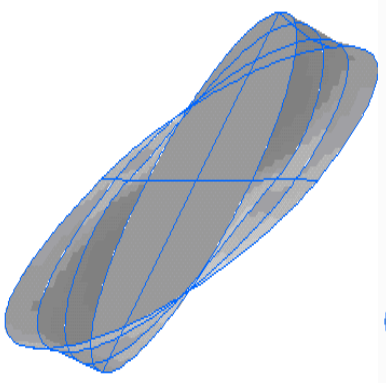

(d)

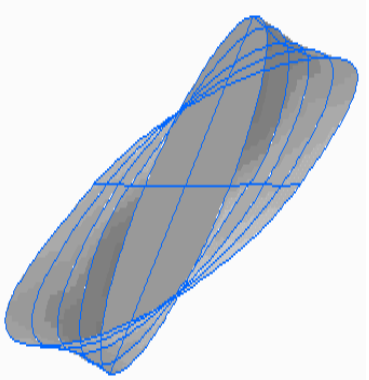

(e)

Fig. 8. Propagation process of initial crack (e.g. $\alpha=45^{\circ}$ case)

\section{Summary}

Researches on mechanisms of 3-D crack development in rock mass have received extensive attention in international communities. Experiments were conducted to investigate the mechanical properties and fracture state of rock-like samples containing single inclined pre-existing crack under uniaxial tension. Test indicates that during the loading and deforming process of samples, elastic deformation takes the most proportion of the whole, and the failure stage is abrupt. Inclination angle of crack has obvious effect on sample strength. With the increase of inclination angle, strength of sample decreases. The growth trace of wing crack is observed on the fracture section.

FRANC3D was adopted to calculate the stress intensity factors along crack contour, and to simulate the crack growth process. Results demonstrate that $K_{\mathrm{I}}$ and $K_{\mathrm{II}}$ values are symmetric to long axis of crack, and $K_{\mathrm{III}}$ are antisymmetric to long axis. $K_{\mathrm{I}}$ increases with the increase of crack inclination angle. For $K_{\text {II }}$ and $K_{\text {III }}$, maximums are reached when $\alpha=45^{\circ}$. Numerical simulation shows that wrapping wing crack and lateral growth due to tensile stress are main propagation mechanism of initial crack growth. Wing crack initiates near the front of long axis, and its growth direction changes from perpendicular to initial crack to horizontal. Lateral growth develops close to the front of short axis, and propagates along the plane of initial crack. Finally, a spatial distortion fracture plane results in the overall failure of sample.

Propagation patterns of 3-D crack under tension are quite different from compressive cases [1], mainly in aspects of growth orientation and velocity. Propagation orientation of wing crack under tension is perpendicular to axial stress, but parallel to axial stress in compressive case. Furthermore, propagation velocity in tensile case is much higher than that in compressive case.

\section{References}

[1] A.V. Dysikin, E. Sahouryeh, R.J. Jewell, H. Joer and K.B. Ustinov: submitted to Engineering Fracture Mechanics (2003).

[2] A.V. Dysikin, L.N. Germanovich, and K.B. Ustinov: submitted to Engineering Fracture Mechanics (1999)

[3] M. Adams, and G. Sines: submitted to Tectonophysics (1978).

[4] R.H.C. Wong, C.M. Law, K.T. Chau, and Wei-Shen Zhu: submitted to International Journal of Rock Mechanics \& Mining Sciences (2004).

[5] Z.Z. Liang, T.H. Yang, C.A. Tang, and J.X. Zhang: submitted to Chinese Journal of Geotechnical Engineering (2005). 